\title{
The Diagnostic Yield and Safety of Ultrasound-Assisted Transthoracic Fine-Needle Aspiration of Drowned Lung
}

\author{
Coenraad F.N. Koegelenberg ${ }^{a}$ Chris T. Bolliger ${ }^{a}$ Elvis M. Irusen ${ }^{a}$ \\ Colleen A. Wright ${ }^{c}$ Mercia Louw ${ }^{c}$ Pawel T. Schubert ${ }^{c}$ Andreas H. Diacon ${ }^{a, b}$

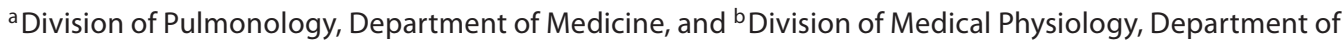 \\ Biomedical Sciences, University of Stellenbosch and Tygerberg Academic Hospital, and 'Division of Anatomical \\ Pathology, Department of Pathology, University of Stellenbosch and National Health Laboratory Service, \\ Cape Town, South Africa
}

\section{Key Words}

Drowned lung $\cdot$ Lung cancer $\cdot$ Transthoracic fine-needle aspiration • Ultrasound

\begin{abstract}
Background: Proximal lung tumors, though not discernable by means of transthoracic ultrasound (US), may cause varying degrees of pulmonary collapse and postobstructive pneumonitis which may give rise to a 'drowned lung' appearance on chest computed tomography (CT) and US. The diagnostic yield for malignancy of US-assisted transthoracic fine-needle aspiration (FNA) of these areas of drowned lung is unknown. Objectives: We aimed to explore the feasibility of US-assisted FNA in this setting by prospectively investigating its diagnostic yield and safety. Methods: We enrolled 31 patients (aged $59.4 \pm 9.7$ years, 17 males) with central tumors and secondary drowned lung on CT scan. A respiratory physician performed transthoracic US to identify the target drowned lung tissue. Three US-assisted superficial FNA passes ( $\leq 20 \mathrm{~mm}$ from the pleura) were followed by 3 deeper FNA passes $(>20 \mathrm{~mm})$ aimed in the direction of a visible or approximated central mass. Rapid on-site evaluation of specimens was used. Results: Superficial FNA was diagnostic in 11
\end{abstract}

patients (35.5\%), whereas deeper FNA was diagnostic in 23 patients $(74.2 \%, p=0.002)$. Deeper FNA confirmed malignancy in all cases with diagnostic superficial FNA. We observed no pneumothoraces or major hemorrhage. All patients were ultimately diagnosed with malignancy (bronchogenic carcinoma, $n=30$; lymphoma, $n=1$ ). Conclusions: US-assisted FNA of drowned lung has an acceptable diagnostic yield and is safe.

Copyright $\odot 2010$ S. Karger AG, Basel

\section{Introduction}

Transthoracic ultrasound (US) has become a valuable diagnostic aid for respiratory physicians [1-6] and is increasingly being used to guide interventional procedures such as thoracentesis and biopsies of the pleura and lung tumors that abut the chest wall [1, 2, 7-9]. Studies have shown that US-assisted transthoracic fine-needle aspiration (FNA) in the setting of intrathoracic malignancies that extend to the chest wall is safe and has a high diagnostic yield, particularly in the setting of bronchogenic carcinoma where the diagnostic sensitivity is greater than $90 \%[7-9]$.

\section{KARGER}

Fax +4161306 1234 E-Mail karger@karger.ch www.karger.com
(C) 2010 S. Karger AG, Basel

0025-7931/11/0811-0026\$38.00/0

Accessible online at:

www.karger.com/res
Dr. Coenraad F.N. Koegelenberg

Division of Pulmonology, Department of Medicine

University of Stellenbosch

PO Box 19063, Tygerberg 7505, Cape Town (South Africa)

Tel. +27 21938 9243, Fax +27 21933 3591, E-Mail coeniefn@sun.ac.za 


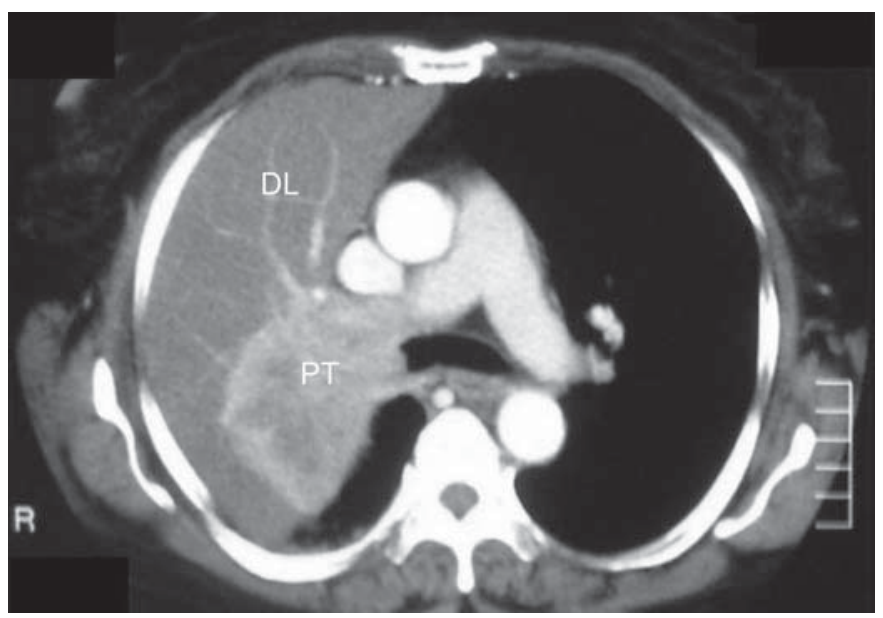

Fig. 1. This CT scan of one of the study subjects shows a distinct central mass lesion (primary tumor) with secondary drowned lung. Note the enhanced pulmonary vasculature. In this particular patient, aspirates were taken in the supine position anterolaterally in order to transect at least $40 \mathrm{~mm}$ of drowned lung (as measured from the visceral pleural to the drowned lung-tumor interface). $\mathrm{PT}=$ Primary tumor; $\mathrm{DL}=$ drowned lung.

The parenchyma of normal aerated lungs is not discernable by means of US. Proximal lung tumors are therefore not considered amenable to US-assisted transthoracic FNA or cutting-needle biopsies. Proximal lung tumors may, however, cause varying degrees of pulmonary collapse (resorptive atelectasis) and postobstructive pneumonitis. 'Drowned lung' is a radiological term often used to describe these areas which are considered to represent accumulated secretions and are typified on chest computed tomography (CT) by enhanced pulmonary vasculature contrasted against the surrounding pulmonary consolidation (fig. 1) [10, 11]. The fluid-filled airways are detectable on US as fluid bronchograms, which are dynamic anechoic tubular structures within consolidated lung that fluctuate with respiration, provided the consolidation extends to the chest wall (fig. 2) $[2,12]$.

The diagnostic yield for malignancy of US-assisted FNA of areas of drowned lung secondary to proximal tumors is not known. Moreover, endobronchial inspection and sampling by means of flexible bronchoscopy is generally considered the standard diagnostic approach in this scenario $[10,13,14]$. We therefore aimed to explore the feasibility of US-assisted transthoracic FNA in this setting by prospectively investigating its diagnostic yield and safety. Furthermore, we tested the hypothesis that

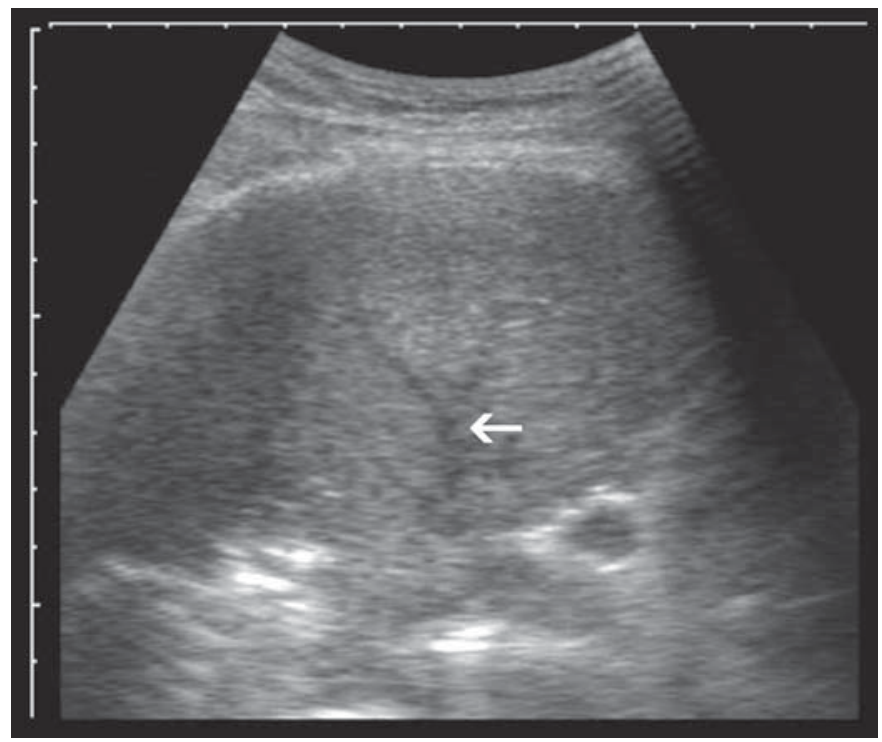

Fig. 2. An example of the US appearance of drowned lung. The arrow depicts the preserved bronchovascular bundle appearing as fluid-filled tubular structures. Note that the fluid bronchograms are best appreciated on real-time US. No clear plane between the drowned lung and tumor mass could be identified in this particular case.

aspirations performed close to the primary tumor would have a greater chance of yielding diagnostic material than more superficial passes.

\section{Materials and Methods}

\section{Study Population}

Our institution is a 1,200-bed academic hospital in Cape Town, South Africa. It is 1 of 2 referral centers and renders a tertiary service to a population of approximately 1.5 million people. Over a 3-year period (from May 2007 to April 2010) we screened the CT scans of all adult patients ( $>18$ years) with suspected bronchogenic carcinoma reviewed at the Division of Pulmonology's daily radiology meeting, which acts as a forum where all internal and external referrals of patients with potential bronchogenic carcinoma are routinely presented (from January 2007 to December 2009,906 cases of confirmed primary lung cancer were presented at this meeting according to our divisional cancer registry). All patients with an unequivocal central mass lesion with secondary drowned lung (on CT scan) were subsequently invited to participate in this prospective observational study, provided that the suspected carcinoma did not extend to the visceral pleura, at least 1 lobe was involved, at least $40 \mathrm{~mm}$ of drowned lung (minimal distance measured from visceral pleura) was present, and a tissue diagnosis was not known. For the purposes of this study, drowned lung was defined on contrasted CT scan as consolidated lung parenchyma with enhanced pulmonary vasculature distinct from and distal to a central mass lesion. The Health Research Ethics 
Committee of Stellenbosch University approved the study (project No. N08/01/010). Written informed consent was obtained from all subjects upon enrolment and also prior to any invasive procedures.

\section{Transthoracic US}

A consultant respiratory physician performed the sonography (Toshiba JustVision 200 SSA-320A; Toshiba Medical Systems Corporation, Utsunomiya, Japan). A standard 3.75- $\mathrm{MHz}$ sector probe was used and the patients' positions for US scanning were determined by the corresponding CT scan, e.g. patients with lower-lobe involvement were generally examined in the sitting position using a bedside table as an armrest, whereas patients with upper-lobe involvement were scanned in the supine position. All procedures were performed in a bronchoscopy suite without the support of a specialist radiologist. The physician was asked to specifically comment on the presence of drowned lung and whether a distinct border between the apparent proximal mass and distal drowned lung could be observed. An associated effusion, if present, was documented as follows: minimal (if the echo-free space was confined to the costophrenic angle), small (if the space was greater than the costophrenic angle but still within the range of the area covered with a $3.75-\mathrm{MHz}$ curvilinear probe), moderate (if the space was greater than a 1-probe range but within a 2-probe range, and large (if the space was larger than a 2-probe range) [1]. The intended puncture site was subsequently identified and marked, and the direction and the depth of interest for the procedure were documented. The site of aspirations was the epicenter of pleural contact, and the intended direction was towards the observed or anticipated location of the central mass lesion while care was taken to avoid any major blood vessels or viscera. All procedures were subsequently performed 'freehand' (not under direct real-time US guidance)

\section{Transthoracic FNA}

Aspirations were performed with 22-gauge spinal needles of 40 - or 90 -mm length as needed (Tae-Chang, Kong Ju City, Korea) connected to a $10-\mathrm{ml}$ syringe under sterile conditions with local anesthesia (lignocaine 1\%) and no sedation. Three superficial passes at a depth not greater than $20 \mathrm{~mm}$ from the visceral pleura were performed, followed by 3 deeper passes ranging from 20 to $60 \mathrm{~mm}$. For the purposes of the study, care was taken not to aspirate pleural fluid or to contaminate the needles with pleural effusion. The inner stylet of the spinal needle was only removed once the target area had been reached, and no suction was applied while the needle was withdrawn. Aspirates (all from slightly different directions and depths) were directly expressed onto slides, smeared, and submitted for rapid on-site evaluation (ROSE) using both Diff-Quik (Rapidiff; Clinical Sciences Diagnostics, Johannesburg, South Africa) and rapid Papanicolaou staining methods [15].

\section{ROSE of Cytology Specimens}

The cytopathologist present in theater was experienced in ROSE and was asked to comment on the presence or absence of diagnostically useful material obtained during each individual pass. Material that was considered not diagnostically useful included exclusively blood, necrotic tissue, or the total absence of cellular material. The cytopathologist was subsequently asked to comment on cytological evidence of drowned lung, which for the purposes of this study was defined as an aspirate that contained a paucity of bronchial cells with a predominance of alveolar macrophages or pneumocytes ( $75 \%$ or more). Finally, he or she was asked to comment on the presence of malignant cells and, if present, whether they could be provisionally categorized as non-small cell lung carcinoma, small cell lung carcinoma, epithelial carcinoma not otherwise specified, or malignant cells of unknown origin.

\section{Immediate Postprocedure Care}

The FNA site was reexamined by means of US immediately after the procedures, and a chest radiograph was obtained if the pre- and postprocedure US findings differed and at the discretion of the attending physician. All patients were observed for at least $2 \mathrm{~h}$ prior to discharge and complications were noted. The presence or absence of chest wall hemorrhage and a pneumothorax was specifically documented.

\section{Further Assessment and Statistical Analysis}

All cytology slides were reviewed in the laboratory by a second experienced cytopathologist who had an array of special stains (including immunohistochemistry) at his or her disposal and had to concur with the original cytopathologist prior to issuing a final cytological diagnosis. In case of disagreement, a third cytopathologist was consulted to resolve the case.

Further investigations for patients who remained undiagnosed or who required additional staging were guided by the patient's attending respiratory physician. These investigations potentially included bronchoscopy with direct forceps and/or transbronchial needle aspiration biopsies (TBNA), CT-guided FNA, or even surgical biopsies. All patients were followed up until a tissue diagnosis could be confirmed. All cases of bronchogenic carcinoma were staged according to the 2002 Union Internationale Contre le Cancer staging system for lung cancer [16].

Descriptive statistics and McNemar's test (performed to assess whether the proportion of positive diagnoses was the same between deep and superficial passes) were utilized. $\mathrm{p} \leq 0.05$ in a 2 -tailed test was considered statistically significant. Unless stated otherwise, data are displayed as means \pm standard deviation (SD). We used standard methods to calculate the sensitivity [17]. We had no firm data to base the sample size estimation of this proof-of-concept study on; therefore, we only used approximations. We considered a difference of $50 \%$ between superficial and deeper passes worth detecting, and posited a yield of 25 and $75 \%$, respectively. A study population of 30 subjects was therefore considered sufficient (with a 5\% confidence level allowing for $25 \%$ nondiagnostic aspirations).

\section{Results}

\section{Patients}

Over the 3-year period we screened $>1,000$ CT scans and identified 39 patients with drowned lung. We enrolled all 31 patients (aged $59.4 \pm 9.7$ years, 17 males) who fulfilled the inclusion criteria. Of these, 30 patients were ultimately diagnosed with bronchogenic carcinoma and 
1 with a lymphoma (tables 1,2$)$. Eight patients were not included as they had an established tissue diagnosis (all bronchogenic carcinoma) at the time of screening. Only 1 of the 31 patients enrolled had undergone a preceding bronchoscopy, which was nondiagnostic (the single case of Hodgkin's lymphoma).

\section{Imaging}

On CT scan the central mass lesions' maximum diameter in any plane ranged from 12 to $60 \mathrm{~mm}$. The extent of pulmonary involvement with regard to drowned lung ranged from 40 to $61 \mathrm{~mm}$ (52.6 $\pm 5.8 \mathrm{~mm})$ expressed as the shortest distance between the pleura and the central mass. Anatomically the areas of drowned lung varied from total lung $(\mathrm{n}=8,25.8 \%)$ to bilobar lung $(\mathrm{n}=1,3.2 \%)$ to unilobar right lung ( $\mathrm{n}=22,71.0 \%)$ (table 3 ).

Drowned lung was confirmed in all cases on US and measured from 40 to $62 \mathrm{~mm}(54.3 \pm 8.9 \mathrm{~mm})$ from the visceral pleura. A clear plain of separation between areas of distal drowned lung and the proximal mass lesion was only observed in 10 cases (32.3\%). Twelve patients (38.7\%) had evidence of an associated effusion ranging from $\mathrm{min}$ imal $(\mathrm{n}=5,16.1 \%)$ to small $(\mathrm{n}=7,22.6 \%)$.

\section{Fine-Needle Aspirations}

A total of 93 superficial FNA passes (range 9-20 mm from the pleura) and 94 deep FNA passes (range 25-60 $\mathrm{mm}$ ) were performed. Laboratory confirmation of bronchogenic carcinoma was possible in 11 of the 31 patients based on specimens obtained by means of superficial passes (sensitivity 35.5\%) and in 23 patients based on specimens obtained with deep FNA passes (sensitivity $74.2 \%, p=0.002$ ). Deep passes confirmed malignancy in all cases in which superficial passes were diagnostic (in no case was a diagnosis made based solely on a superficial FNA). Cytological evidence of drowned lung was present in 28 patients based on superficial passes $(90.3 \%)$ and in 24 patients based on deep passes $(77.4 \%$, $\mathrm{p}=0.343)$.

At least 1 of the 3 deep FNA passes was performed to a depth greater than that of the measured (on US) or estimated (on CT scan) tumor-drowned lung interface in 8 patients. These passes yielded the only positive specimens in 3 patients, whereas both sets (including passes that did not cross this interface) were positive in 3 more cases. Both were negative in 2 cases.

\section{Further Investigations}

Of the 23 patients with a firm cytological diagnosis of bronchogenic carcinoma, most had either advanced non-
Table 1. Final established diagnoses of all study subjects $(n=31)$

\begin{tabular}{lrr}
\hline Diagnoses & $\mathrm{n}$ & $\%$ \\
\hline Non-small cell lung cancer & & \\
$\quad$ Adenocarcinoma & 10 & 32.3 \\
$\quad$ Squamous cell carcinoma & 12 & 38.7 \\
$\quad$ Undifferentiated/large cell & 3 & 9.7 \\
Small cell lung cancer & 5 & 16.1 \\
Hodgkin's lymphoma (nodular sclerosing type) & 1 & 3.2 \\
\hline
\end{tabular}

Table 2. Final TNM staging ${ }^{1}$ of all cases of non-small cell lung cancer $(\mathrm{n}=25)$

\begin{tabular}{lcc}
\hline Stage & $\mathrm{n}$ & $\%$ \\
\hline I & 0 & 0 \\
II & 2 & 8 \\
IIIA & 2 & 8 \\
IIIB & 10 & 40 \\
IV & 11 & 44 \\
\hline
\end{tabular}

${ }^{1}$ According to the 2002 Union Internationale Contre le Cancer staging system [16].

Table 3. Anatomical extent of drowned lungs $(n=31)$

\begin{tabular}{lrr}
\hline Lobes involved & $\mathrm{n}$ & $\%$ \\
\hline Complete right lung & 5 & 16.1 \\
Isolated right upper lobe & 11 & 35.5 \\
Isolated right middle lobe & 1 & 3.2 \\
Isolated right lower lobe & 1 & 3.2 \\
Right lower and middle lobes & 1 & 3.2 \\
Complete left lung & 3 & 9.7 \\
Isolated left upper lobe & 9 & 29.0 \\
Isolated left lower lobe & 0 & 0.0 \\
\hline
\end{tabular}

small cell lung cancer (table 2) or small cell lung cancer. Only 3 patients required a subsequent bronchoscopy with TBNA to clarify the staging. Of the 8 cases in which a diagnosis could not be established by means of US-assisted transthoracic FNA, 7 were diagnosed by bronchoscopy with TBNA and 1 by mediastinoscopy (the solitary case of lymphoma). In total, 11 patients (35.5\%) required further investigation, which in no case was deemed high risk. 


\section{Complications}

The procedures were well tolerated and no pneumothoraces were noted. Only 1 case of minor hemorrhage which subsided upon compression alone and did not require surgical sutures was documented.

\section{Discussion}

This prospective study is, to the best of our knowledge, the first to investigate US-assisted transthoracic FNA of drowned lung to determine the diagnostic yield for malignancy. We found that FNA passes at a depth greater than $20 \mathrm{~mm}$ from the visceral pleura had a diagnostic sensitivity of $74.2 \%$ and that they were more likely to contain malignant cells than more superficial passes (74.2 vs. $35.5 \%, p=0.002)$. Furthermore, we observed no serious complications.

We previously showed that transthoracic US-assisted FNA with ROSE and cutting-needle biopsies had a combined sensitivity of $81 \%$ for a spectrum of benign and malignant lesions that abutted the pleura [8]. More importantly, this technique had a sensitivity of $95 \%$ for bronchogenic carcinoma and a pneumothorax rate of only $1.3 \%$ [8]. In another study we found that this modality had a diagnostic sensitivity of $96 \%$ in patients who presented with superior vena cava syndrome with an associated mass lesion abutting the chest wall [9]. Yang et al. [18] previously found that US-guided cutting-needle biopsies have a diagnostic yield of $94.6 \%$ for subpleural pulmonary tumors. Although the diagnostic yield in the present study is certainly not on par with these figures, our data provides novel evidence that transthoracic US-assisted FNA may even be useful in cases where the primary tumor does not extend to the chest wall.

Approximately $35 \%$ of patients had cytological evidence of bronchogenic carcinoma on superficial FNA passes. Although this figure was significantly less than on deeper passes, it remained an important observation as these aspirates were obtained at a distance from the associated pulmonary mass. Interestingly this yield is very similar to that of sputum cytology $[13,19,20]$, suggesting that endobronchial shedding and pooling of tumor cells may be the source of the diagnostic material obtained by means of superficial sampling from the drowned lung. We specifically aimed to avoid the potential contamination of specimens with malignant cells inadvertently obtained from pleural fluid, making it a much less likely source.
Bronchoscopy has an overall sensitivity of $88-95 \%$ for malignancy in patients with centrally located lung lesions $[13,21]$, which is superior to the sensitivity of US-assisted transthoracic FNA. It allows for the inspection of central airways, evaluation for therapeutic airway management, and the acquisition of histological samples that may allow for superior subtyping and immunohistochemistry [13]. Furthermore, mediastinal nodal staging by means of TBNA has important implications for the staging and management of lung cancer $[16,22]$. Ten patients underwent bronchoscopy in our study, which in 3 patients only indicated the need for endobronchial mediastinal staging. In fact, the vast majority of our study population had advanced lung cancer.

As this study was purely a proof-of-concept study designed to explore the feasibility of US-assisted transthoracic FNA in a novel setting, it was not designed to compare US-assisted transthoracic FNA with bronchoscopy (or CT-guided FNA) or to define which patient may be best suited for a particular procedure. Notwithstanding these important considerations, our findings suggest that transthoracic US-assisted FNA of drowned lung may be utilized in specific circumstances, which may include patients who are considered high risk for bronchoscopy, those who refuse to undergo bronchoscopy, and those in whom a preceding bronchoscopy had been nondiagnostic. Moreover, it may be viewed as an acceptable alternative when endoscopic mediastinal staging is irrelevant [1] or when bronchoscopy is unavailable. Only future randomized studies will, however, be able to specifically delineate the subgroup of patients with drowned lung in whom a bronchoscopy may be considered superfluous.

Some practical considerations need to be emphasized. US-assisted FNA can be performed by a single clinician with no sedation and minimal monitoring, even potentially outside of theater [1]. The disposables (especially the needles) are cheap, and we once again showed that the procedure is safe even without the assistance of specialist radiologists. We observed no pneumothoraces or major hemorrhage, a finding that was not unexpected as no aerated lung was transversed and only 22 -gauge needles were utilized.

We specifically only included cases with at least 40 $\mathrm{mm}$ of drowned lung (as measured from the visceral pleura) in order to have ample drowned lung to sample and to avoid direct sampling of the primary tumor by means of superficial FNA passes. A clear plane between the drowned lung and tumor mass could, however, only be identified in a minority of subjects with the aid of US, and in the majority this plane was purely an estimation 
based on CT scan findings. The relative contribution of FNA passes from the actual tumor mass and from surrounding drowned lung to the reported diagnostic yield for deep passes is therefore uncertain. Although this may be viewed as a limitation of the study, this distinction seems irrelevant, as in practice knowing which specimen confirmed a cytological diagnosis of bronchogenic carcinoma would seem immaterial.

Our study was not designed to establish the true incidence of drowned lung secondary to lung cancer in our population, but our data suggest it to be a relatively rare presentation. Over a 3-year period we identified only 39 patients with central tumors and drowned lung, 38 of whom were diagnosed with lung cancer. We recorded 906 cases of primary lung cancer during that period, suggesting that approximately $4 \%$ of all patients with lung cancer had drowned lung at the time of initial presentation.

In conclusion, US-assisted FNA of drowned lung is safe and has an acceptable diagnostic yield, particularly when deep passes are performed. It may be a feasible al- ternative to bronchoscopy where endoscopy is considered high risk, where bronchoscopy has failed to yield a diagnosis, or where mediastinal staging is considered unnecessary. Future studies aimed at specifically delineating the subgroup of patients in whom a bronchoscopy may be considered superfluous are therefore indicated.

\section{Acknowledgements}

The authors would like to thank Professor Martin Kidd (Centre for Statistical Consultation, University of Stellenbosch) for his assistance with the statistical analysis of the study data. The US equipment was acquired with funds donated by the Holland Stellenbosch Medical Foundation.

\section{Financial Disclosure and Conflicts of Interest}

The authors report no conflicts of interest.

\section{References}

1 Koegelenberg CFN, Bolliger CT, Diacon AH: Pleural ultrasound; in Light RW, Lee YC (eds): Textbook of Pleural Disease, ed 2. London, Hodder \& Stoughton, 2008, pp 275-283.

2 Koegelenberg CFN, Diacon AH, Bolliger CT: Transthoracic ultrasound of the chest wall, pleura, and the peripheral lung; in Bollger CT, Herth FJF, Mayo PH, Miyazama T, Beamis JF (eds): Progress in Respiratory Research. Clinical Chest Ultrasound. Basel, Karger, 2009, vol 37, pp 22-33.

>3 Beckh S, Bolcskei PL, Lessnau KD: Real-time chest ultrasonography: a comprehensive review for the pulmonologist. Chest 2002;122: 1759-1773.

4 Diacon AH, Theron J, Bolliger CT: Transthoracic ultrasound for the pulmonologist. Curr Opin Pulm Med 2005;11:307-312.

$\checkmark 5$ Mayo PH, Doelken P: Pleural ultrasonography. Clin Chest Med 2006;27:215-217.

$\checkmark 6$ Evans AL, Gleeson FV: Radiology in pleural disease: state of the art. Respirology 2004;9: 300-312.

$\checkmark 7$ Diacon AH, Schuurmans MM, Theron J, Wright CA, Bolliger CT: Safety and yield of ultrasound-assisted transthoracic biopsy performed by pulmonologists. Respiration 2004;71:519-522.
$>8$ Diacon $\mathrm{AH}$, Theron J, Schubert $\mathrm{P}$, Brundyn K, Louw M, Wright CA, Bolliger CT: Ultrasound-assisted transthoracic biopsy: fineneedle aspiration or cutting-needle biopsy? Eur Respir J 2007;29:357-362.

$\checkmark 9$ Koegelenberg CF, Bolliger CT, Plekker D, Wright CA, Brundyn K, Louw M, Schubert P, van den Heuvel MM, Diacon AH: Diagnostic yield and safety of ultrasound-assisted biopsies in superior vena cava syndrome. Eur Respir J 2009;33:1389-1395.

10 Venta LA, Shapir J: Enhancement of pulmonary vasculature in pulmonary consolidation as seen by computed tomography. J Comput Tomogr 1985;9:133-135.

$>11$ Nadig SN, Block MI: 'Drowned lung' following lobectomy and radiation therapy: a case report. JSC Med Assoc 2003;99:26-29.

12 Lichtenstein DA, Mezière GA: Relevance of lung ultrasound in the diagnosis of acute respiratory failure: the BLUE protocol. Chest 2008;134:117-125.

13 Rivera MP, Mehta AC: Initial diagnosis of lung cancer. ACCP evidence-based clinical practice guidelines (2nd edition). Chest 2007;132:131S-148S.

14 Medford AR, Agrawal S, Free CM, Bennett JA: A prospective study of conventional transbronchial needle aspiration: performance and cost utility. Respiration 2010;79: 482-489.
15 Keebler KM: Cytopathology techniques; in Bibbo M (ed): Comprehensive Cytopathology, ed 2. Philadelphia, W.B. Saunders, 1997, pp 881-906.

16 Union Internationale Contre le Cancer: TNM classification of malignant tumours, ed 6. New York, Wiley-Liss, 2002, p 272.

17 Fisher L, van Belle G: Biostatistics: A Methodology for the Health Sciences. New York, Wiley, 1993, p 206.

18 Yang PC, Luh KT, Chang DB, Yu CJ, Kuo SH, Wu HD: Ultrasound-guided core biopsy of thoracic tumors. Am Rev Respir Dis 1992; 146:763-767

19 Petty TL: The early identification of lung carcinoma by sputum cytology. Cancer 2001;89: 2461S-2464S

$>20$ Gledhill A, Bates C, Henderson D, DaCosta P, Thomas G: Sputum cytology: a limited role. J Clin Pathol 1997;50:566-568.

21 Gasparini S, Ferretti M, Secchi EB, Baldelli S, Zuccatosta L, Gusella P: Integration of transbronchial and percutaneous approach in the diagnosis of peripheral pulmonary nodules or masses: experience with 1,027 consecutive cases. Chest 1995;108:131-137.

22 Detterbeck FC, Boffa DJ, Tanoue LT: The new lung cancer staging system. Chest 2009; 136:260-271. 